\title{
Efecto de las estatinas en el desarrollo de síndrome postrombótico: estudio de cohorte
}

\author{
Effect of statins on development of post thrombotic syndrome: cohort study \\ Héctor J. Peroni*, María F. Grande-Ratti, Fernando J. Vázquez, Fernán B. González de Quirós, \\ María L. Posadas-Martínez y Diego H. Giunta \\ Servicio de Clínica Médica, Hospital Italiano de Buenos Aires, Buenos Aires, Argentina
}

\begin{abstract}
Resumen
Objetivo: Explorar la asociación entre consumo de estatinas (CE) y desarrollo de síndrome postrombótico (SPT). Método: Cohorte retrospectiva con pacientes con primer episodio de trombosis venosa profunda (TVP) entre el 06/2006 y el 12/2017, incluidos en el Registro Institucional de Enfermedad TromboEmbólica (RIET) del Hospital Italiano de Buenos Aires. Se consideró exposición al CE entre los 30 días previos y hasta 180 días posterior al diagnóstico de TVP. Se definió SPT según constaba este dato en la base de seguimiento del RIET. Se evaluó el desarrollo de SPT con un modelo de riesgos proporcionales de Cox, reportando hazard ratios (HR) crudas y ajustadas. Se consideró la confusión por indicación del CE y se utilizó un propensity score (PS) para el ajuste del riesgo estimado, reportando los HR con sus intervalos de confianza del 95\% (IC 95\%). Resultados: Se incluyeron 905 pacientes, de los cuales 273 fueron CE y 632 no consumidor de estatinas (NCE). Al seguimiento, la incidencia de SPT fue: 6.59\% (18) en el grupo CE y 8.07\% (51) en el grupo NCE, con $p=0.412$. La razón de riesgo para el desarrollo de SPT de CE resultó no significativa (HR cruda: 0.78; IC 95\%: 0.43$1.41 ; p=0.414)$. La HR de CE ajustada por edad, sexo, antiinflamatorios no esteroideos, corticosteroides, inmovilidad, anticoagulante, hipertensión arterial, diabetes, dislipidemia, insuficiencia renal crónica, enfermedad coronaria, accidente cerebrovascular, insuficiencia cardiaca y enfermedad oncológica fue 0.45 (IC 95\%: 0.13-1.5; $p=0.196$ ). La HR del CE ajustado por edad, sexo, antiinflamatorios no esteroideos, corticosteroides, inmovilidad, tratamiento anticoagulante, enfermedad oncológica y PS fue de 0.52 (IC 95\%: 0.17-1.66; $p=0.272$ ). Conclusiones: EI CE no se asoció con menor SPT, aunque hubo escaso número de eventos detectados.
\end{abstract}

Palabras clave: Trombosis venosa. Inhibidores de hidroximetilglutaril-CoA reductasa. Síndrome postrombótico. Epidemiología.

\begin{abstract}
Objective: To evaluate the association between statin consumption and development of post-thrombotic syndrome (PTS). Methods: Retrospective cohort study which included patients with a first episode of deep vein thrombosis (DVT) between 06/2006 and 12/2017, included in the Institutional Registry of ThromboEmbolic Disease of the Italian Hospital of Buenos Aires, Argentina. Exposure to statin use (SU) was considered between the 30 days before and up to 180 days after the diagnosis
\end{abstract}


of DVT. PTS was defined as recorded dataset on registry. The development of PTS was evaluated with Cox proportional hazards model, raw and adjusted hazard ratios (HR) were reported. Confusion was considered by indication of SU and a propensity score (PS) was used for adjustment. We reported $H R$ with their $95 \%$ confidence interval (Cl); $p$ value $<0.05$ was considered statistically significant. Results: Of 1393 patients, 905 were included for the analysis, of which 273 were SU and 632 non-statin users (NSU). At follow-up, incidence of PTS was: $6.59 \%$ (18) in the SU group and $8.07 \%$ (51) in the NSU group, with $p=0.412$. Crude HR for PTS for SU was not significant $(0.78 ; 95 \% \mathrm{Cl}: 0.43-1.41 ; p=0.414)$. Adjusted HR of SU by age, sex, non-steroidal anti-inflammatory drugs, corticosteroids, immobility, anticoagulant, high blood pressure, diabetes, dyslipidemia, chronic renal failure, coronary heart disease, stroke, heart failure and cancer disease was 0.45 (95\% Cl: 0.13 1.5; $p=0.196)$ for PTS. While HR for the development of PTS adjusted by age, sex, non-steroidal anti-inflammatory drugs, corticosteroids, immobility, anticoagulant treatment, cancer disease and PS of the SU was 0.52 (95\% Cl: 0.17-1.66; $p=0.272$ ).

Conclusion: No statistically significant association was found between CE and the development of SPT, although there were a small number of events detected in both groups.

Key words: Venous thrombosis. Hydroxymethylglutaryl-CoA reductase inhibitors. Post-thrombotic syndrome. Epidemiology.

\section{Introducción}

La trombosis venosa profunda (TVP) es una enfermedad que presenta una incidencia anual de un caso cada 1,000 habitantes-año $0^{1,2}$. Su evolución puede predisponer a diferentes complicaciones como tromboembolia pulmonar (TEP), hemorragias, TVP recurrente y/o desarrollo de graves secuelas postrombóticas ${ }^{3}$.

Una de las complicaciones crónicas más relevantes es el síndrome postrombótico (SPT), que se caracteriza clínicamente por dolor crónico, edema y pesadez de la extremidad afectada. En casos severos pueden desarrollarse úlceras venosas. EI SPT se manifiesta entre el 17 y el $35 \%$ dentro del primer año, llegando a tener una incidencia acumulada a los dos años del $50 \%{ }^{4}$.

Se conoce que el SPT es el resultado de la hipertensión venosa que sigue al desarrollo de la incompetencia valvular (consecuencia del daño sufrido por las válvulas venosas en el momento del episodio trombótico agudo y la fase de recanalización venosa), al reflujo y a la obstrucción venosa. En estas fases actúan diferentes componentes inflamatorios que representan uno de los factores determinantes del desarrollo de $\mathrm{SPT}^{5}$. En este contexto, las estatinas podrían ayudar a disminuir la inflamación crónica por su efecto pleiotrópico y antiinflamatorio, generada al actuar sobre la regulación, la disminución en la expresión y secreción de diferentes factores proinflamatorios ${ }^{6,7}$. De esta manera, podrían disminuir el desarrollo consiguiente de SPT en los pacientes que hayan tenido una TVP8.

Debido a que el SPT es generado en gran medida por el proceso inflamatorio que se genera en las venas afectadas por la TVP y como las estatinas presentan un potente efecto pleiotrópico que actúa disminuyendo la inflación, nos preguntamos si existe asociación entre el consumo de estatinas (CE) y el desarrollo de SPT luego del primer episodio de TVP.

\section{Materiales y métodos}

Se diseñó una cohorte retrospectiva conformada por pacientes adultos con un primer episodio de TVP, incluidos en el Registro Institucional de Enfermedad TromboEmbólica (RIET) del Hospital Italiano de Buenos Aires (HIBA) entre junio de 2006 y diciembre de 2017. Se excluyeron los pacientes que se negaron al consentimiento informado oral del RIET y aquellos que no tuvieran seguimiento telefónico.

EI HIBA es un hospital universitario de alta complejidad de la Ciudad Autónoma de Buenos Aires que funciona como una red integrada de salud en Argentina. Cuenta con 18 centros de atención ambulatoria, con aproximadamente 750 camas. En su sistema de atención, ofrece servicios médicos integrales a una población de 150,000 afiliados a la prepaga institucional (Plan de Salud del Hospital Italiano [PSHIBA]), que garantiza la atención en forma exclusiva y completa dentro de este sistema. Toda la información se centraliza en una historia clínica electrónica (HCE) única9,10, que concentra toda la información administrativa (p. ej., estudios, solicitud de turnos, turnos ambulatorios, internaciones, consumo de fármacos) y toda su información clínica (problemas de salud, diagnósticos clínicos, evoluciones médicas, resultados de laboratorios y estudios, entre otros). Los problemas de salud o comorbilidades son codificados automáticamente mediante un servidor de terminología con tesauro local que mapea y codifica la información con el vocabulario controlado SNOMED-CT ${ }^{11-14}$.

Todas las variables de este estudio fueron recolectadas de la información contenida en la evaluación 
sistemática del RIET, representando una base de datos secundaria de alta calidad. EI RIET funciona desde el 2006, como un registro prospectivo de casos incidentes de enfermedad tromboembólica venosa (ETV) diseñado y conducido por el Área de Investigación en Medicina Interna del Servicio de Clínica Médica. En el mismo se incluyen pacientes correspondientes a todas las coberturas médicas (no restringido a PSHIBA). Las TVP se diagnostican por ecografía Doppler venoso de miembros inferiores. Las venas incluidas fueron: plexo sóleo o venas gemelares con afección del cayado, venas tibiales posteriores y anteriores, vena poplítea, vena femoral (femoral superficial), cayado de la safena, vena ilíaca externa, vena glútea inferior y superiores, y vena ilíaca interna.

A los fines de este estudio, el grupo expuesto estuvo constituido por pacientes que se encontraron consumiendo estatinas ${ }^{15}$ : atorvastatina, rosuvastatina, simvastatina, pravastatina, fluvastatina y lovastatina. Se definió consumidor de estatina (CE) aquel paciente que posee registro de compra en farmacia y/o posee registro en la HCE de estatinas dentro de los 30 días antes y hasta 180 días posterior al diagnóstico del primer episodio de TVP.

Todos los pacientes fueron seguidos de manera prospectiva mediante la revisión sistemática de la HCE y la información obtenida por medio del seguimiento telefónico sistemático del RIET. El inicio del seguimiento fue la fecha índice del primer episodio de TVP. Se los siguió hasta la fecha de: muerte, pérdida de seguimiento o fin administrativo del estudio.

Se recolectaron los eventos SPT con estrategia múltiple, mediante: a) adaptación del cuestionario de Villalta en las evaluaciones telefónicas ${ }^{16}$, y b) revisión de HCE (para detectar problema codificado o diagnóstico clínico). Se detectaron los casos de fallecimiento y sus fechas utilizando bases de datos secundarias (bases de internaciones para detectar las muertes intrahospitalarias) y captura primaria (la baja por fallecimiento desde el padrón, dato restringido únicamente a pacientes del PSHIBA; y la reportada por familiares en el seguimiento telefónico, independiente de la cobertura).

Según el cálculo de tamaño muestral, para una potencia del $80 \%$, con una probabilidad de error alfa de aproximadamente un $5 \%$ y un test bilateral, se requieren mínimo 100 pacientes en cada grupo, asumiendo una incidencia de 0.5 para el desarrollo del SPT en el grupo de no consumidores de estatinas (NCE) y del 0.3 para el grupo de CE. A su vez, se evaluó la asociación entre CE y SPT ajustado por propensity score (PS). Para evaluar esta asociación, se requieren 10 eventos por variable incluida en el modelo. Considerando un modelo multivariante, con al menos seis variables, se requieren 60 eventos de SPT.

La PS se realizó con la intención de resolver el natural sesgo de confusión por indicación del CE debido al diseño observacional sin aleatorización de la exposición. Teniendo en cuenta que la población general presenta una alta tasa de mortalidad y pudiendo interferir esta con el desarrollo de SPT, realizamos un análisis de sensibilidad con modelos de regresión que consideran eventos competitivos. Se utilizaron modelos de regresión de Fine y Gray ${ }^{17,18}$ considerando al fallecimiento como evento competitivo. Las variables utilizadas para la PS fueron las siguientes: edad (odds ratio [OR]: 1.01; IC 95\%: 1.00-1.03), sexo femenino (OR: 0.88; IC 95\%: 0.61-1.25), hipertensión arterial (HTA) (OR: 0.86; intervalo de confianza [IC] 95\%: 0.571.30), enfermedad coronaria (OR: 2.26; IC 95\%: 1.333.84), diabetes (DBT) (OR: 1.30; IC 95\%: 0.78-2.14), dislipidemia (DLP) (OR: 8.76; IC 95\%: 5.91-12.97), accidente cerebrovascular (ACV) (OR: 1.78; IC 95\%: 1.072.96), insuficiencia cardiaca congestiva (ICC) (OR: 1.09; IC 95\%: 0.65-1.82) e insuficiencia renal crónica (IRC) (OR: 0.87; IC 95\%: 0.52-1.45). Se presentan las subhazard ratios (sHR) con sus IC 95\%.

Se describieron las variables cuantitativas como media y desviación estándar (DS) o mediana e intervalo intercuartílico (RIC), según distribución observada y las variables categóricas como frecuencia absoluta y porcentaje. Se compararon las proporciones entre ambos grupos con el test de chi cuadrado o exacto de Fisher, según supuestos. Se compararon las medias con la t test y las medianas con el test de Mann-Whitney. Para evaluar la hipótesis principal del estudio, se estimó el tiempo al evento del desarrollo de SPT en ambos grupos con el método de Kaplan-Meier. Para esto, se consignaron las siguientes fechas: primer evento de TVP, último seguimiento, SPT y fallecimiento. Se consideró como fecha de fin de seguimiento lo que ocurriera primero: el desarrollo del SPT, la muerte o la fecha de último contacto. El tiempo al evento para ambas variables dependientes se evaluó en meses. Se estimaron las densidades de incidencias en ambos grupos y se presentaron con sus IC 95\% por cada 100 personas/ año. Se estimaron las razones de incidencias entre CE y NCE, y se presentaron con sus IC $95 \%$. Se estimaron los HR con sus IC 95\%: utilizando un modelo de regresión de riesgos proporcionales de Cox bivariado. Se consideraron como potenciales confundidores las variables estadísticamente asociadas al desarrollo del SPT y aquellas consideradas de importancia clínica 
Tabla 1. Comparación entre incluidos vs. excluidos (por falta de seguimiento)

\begin{tabular}{|l|c|c|c|}
\hline & Incluidos (n= 905) & Excluidos (n= 488) & p valor \\
\hline Embolia pulmonar concomitante a la TVP & $20.77 \%(188)$ & $21.11 \%(103)$ & 0.884 \\
\hline Estatina & $30.17 \%(273)$ & $16.39 \%(80)$ & 0.001 \\
\hline Edad, en años* & $78(16)$ & $76(16)$ & 0.001 \\
\hline Sexo femenino & $63.54 \%(575)$ & $60.25 \%(294)$ & 0.227 \\
\hline Índice de masa corporal & & \\
\hline Comorbilidades & $27.28(6.02)$ & $27.42(5.42)$ & 0.675 \\
\hline Score de Charlson* & & & \\
\hline Tabaquismo & $2(3)$ & $2(4)$ & 0.417 \\
\hline Hipertensión arterial & $6.74 \%(61)$ & $7.17 \%(35)$ & 0.761 \\
\hline Enfermedad coronaria & $61.55 \%(577)$ & $65.16 \%(318)$ & 0.183 \\
\hline Diabetes & $9.06 \%(82)$ & $7.58 \%(37)$ & 0.346 \\
\hline Dislipidemia & $10.28 \%(93)$ & $12.70 \%(62)$ & 0.169 \\
\hline Accidente cerebro vascular & $45.41 \%(411)$ & $47.13 \%(230)$ & 0.540 \\
\hline Insuficiencia cardiaca & $9.72 \%(88)$ & $5.94 \%(29)$ & 0.015 \\
\hline Insuficiencia renal crónica & $9.94 \%(90)$ & $10.66 \%(52)$ & 0.676 \\
\hline Enfermedad oncológica & $10.94 \%(99)$ & $8.61 \%(42)$ & 0.168 \\
\hline EPOC & $28.84 \%(261)$ & $33.40 \%(163)$ & 0.078 \\
\hline Consumo de AlNE & $10.72 \%(97)$ & $11.07 \%(54)$ & 0.842 \\
\hline
\end{tabular}

*Mediana (rango intercuartílico).

${ }^{\dagger}$ Media ( \pm desviación estándar).

TVP: trombosis venosa profunda; EPOC: enfermedad pulmonar obstructiva crónica; AINE: antiinflamatorios no esteroideos.

como confundidores. Se utilizó un modelo de riesgos proporcionales de Cox multivariado para ajustar por estos potenciales confundidores. Las variables confusoras utilizadas para el ajuste fueron la siguientes: edad, sexo, antiinflamatorios no esteroideos (AINE), corticosteroides, inmovilidad, tratamiento anticoagulante, HTA, DBT, DLP, IRC, enfermedad coronaria, ACV, ICC y enfermedad oncológica. Se consideró estadísticamente significativa una $p<0.05$. Se utilizó el software estadístico STATA 13.

El estudio se llevó a cabo en total acuerdo con la normativa ética nacional e internacional vigente. Todos los datos del estudio fueron tratados con máxima confidencialidad. El protocolo de este proyecto fue evaluado y aprobado por el comité de ética institucional (número 2453).

\section{Resultados}

De un total de 1,393 pacientes potencialmente incluibles, con primer episodio de TVP confirmada e incluidos en el RIET, 488 fueron excluidos debido a la falta de seguimiento telefónico o fallecimiento en el mismo episodio (Tabla 1). Se incluyeron 905 pacientes para el análisis, de los cuales 273 fueron CE y 632 NCE (Fig. 1).
Con respecto a las diferencias basales entre los pacientes CE y NCE, las diferencias estadísticamente significativas fueron: mayor edad (77.46 vs. 73.65 años; $p<0.001$ ), mayor tasa de HTA (76.92 vs. $54.91 \%$; $p<0.001$ ), de enfermedad coronaria (18.32 vs. $5.06 \%$; $p<0.001$ ), de DBT (16.12 vs. $7.75 \% ; p<0.001$ ), de dislipemia (80.95 vs. $30.06 \%$; $p<0.001)$, de ACV (17.58 vs. $6.33 \% ; p<0.001)$, de insuficiencia cardiaca (15.02 vs. $7.75 \%$; $p<0.001)$, de IRC (14.29 vs. $9.49 \%$; $\mathrm{p}=0.034)$ y de consumo previo de AINE (28.94 vs. $21.04 \% ; p=0.010$ ), en todos los resultados de los CE con respecto a los de los NCE respectivamente. En la tabla 2 se presentan todas las características evaluadas y la comparación entre CE y NCE.

La mayoría de los pacientes $(51 \%)$ recibía atorvastatina. La tabla 3 muestra detalles sobre sobre el CE, con las frecuencias de cada fármaco y las dosis.

El grupo CE tuvo mayor tasa de anticoagulación comparado con el grupo NCE (91 vs. $86 \% ; p=0.036$ ). El tipo de anticoagulante más prescrito fue antagonistas de la vitamina $\mathrm{K}(\mathrm{AVK})$. Menos del $1 \%$ en ambos grupos recibieron nuevos anticoagulantes orales (NOA). El $91.21 \%$ (249) de los CE se encontraban anticoagulados $(50.92 \%$ con heparina de bajo peso molecular, $54.21 \%$ con AVK y $0.37 \%$ con NOA), mientras que el $86.23 \%$ (545) del grupo NCE se encontraba anticoagulado $(49.37 \%$ con heparina de bajo peso 


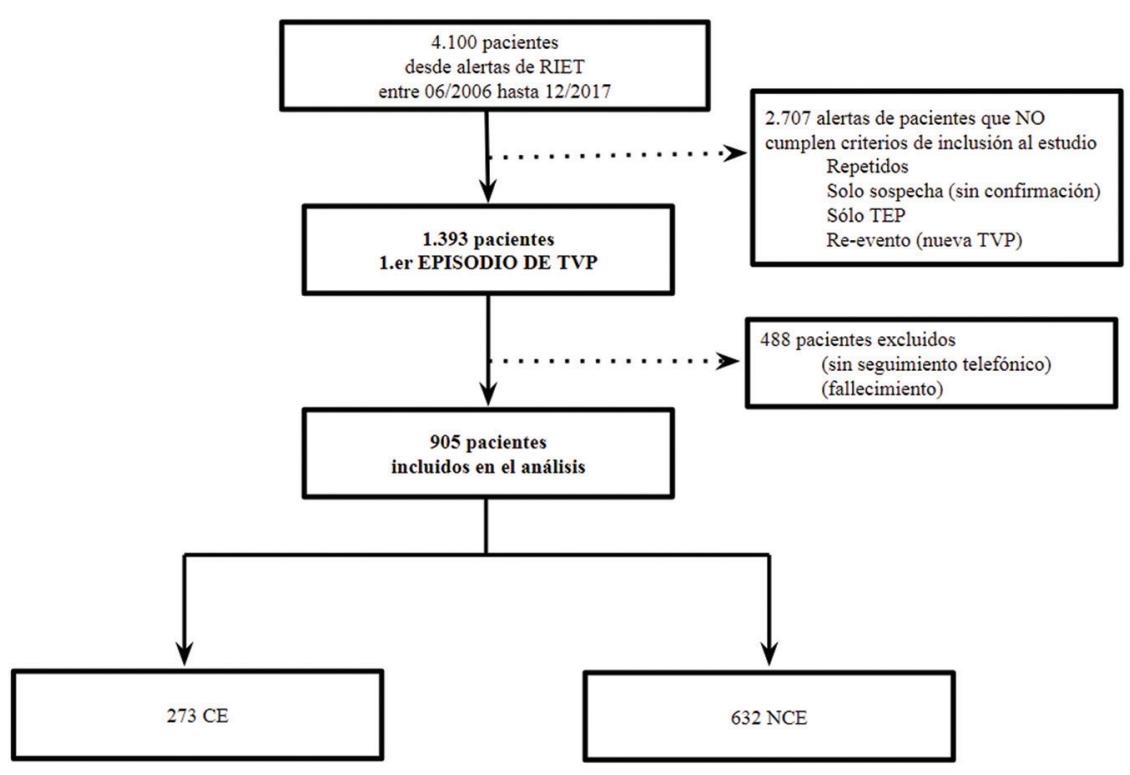

Figura 1. Diagrama del flujo de inclusión de los pacientes en el estudio.

RIET: Registro Institucional de Enfermedad TromboEmbólica; TEP: tromboembolia pulmonar; CE: consumidores de estatinas; NCE: no consumidores de estatinas; TVP: trombosis venosa profunda.

Tabla 2. Características basales de los pacientes incluidos con TVP por grupo CE o NCE

\begin{tabular}{|c|c|c|c|}
\hline & CE (n = 273) & NCE $(n=632)$ & $\mathbf{p}$ \\
\hline $\begin{array}{l}\text { Relacionadas al paciente } \\
\text { Edad, en años* } \\
\text { Sexo femenino } \\
\text { Índice de masa corporal* }\end{array}$ & $\begin{array}{c}77.46(9.68) \\
59.34 \%(162) \\
27.43(5.64)\end{array}$ & $\begin{array}{c}73.65(15.33) \\
65.35 \%(413) \\
27.21(6.19)\end{array}$ & $\begin{array}{c}<0.001 \\
0.085 \\
0.334\end{array}$ \\
\hline $\begin{array}{l}\text { Comorbilidades } \\
\text { Tabaquismo } \\
\text { Hipertensión arterial } \\
\text { Enfermedad coronaria } \\
\text { Diabetes } \\
\text { Dislipidemia } \\
\text { Accidente cerebrovascular } \\
\text { Insuficiencia cardiaca } \\
\text { Insuficiencia renal crónica } \\
\text { Inmovilidad } \\
\text { Enfermedad oncológica } \\
\text { EPOC } \\
\text { Consumo de AINE } \\
\text { Consumo de corticosteroides } \\
\text { Consumo de cilostazol }\end{array}$ & $\begin{array}{c}5.86 \%(16) \\
76.92 \%(210) \\
18.32 \%(50) \\
16.12 \%(44) \\
80.95 \%(221) \\
17.58(48) \\
15.02 \%(41) \\
14.29 \%(39) \\
61.90 \%(169) \\
25.27 \%(69) \\
13.55 \%(37) \\
28.94 \%(79) \\
24.91 \%(68) \\
2.93 \%(8)\end{array}$ & $\begin{array}{c}7.12 \%(45) \\
54.91 \%(347) \\
5.06 \%(32) \\
7.75 \%(49) \\
30.06 \%(190) \\
6.33 \%(40) \\
7.75 \%(49) \\
9.49 \%(60) \\
57.28 \%(362) \\
30.38 \%(192) \\
9.49 \%(60) \\
21.04 \%(133) \\
24.84 \%(157) \\
1.27 \%(8)\end{array}$ & $\begin{aligned} & 0.488 \\
&< 0.001 \\
&< 0.001 \\
&< 0.001 \\
&< 0.001 \\
&< 0.001 \\
&< 0.001 \\
& 0.034 \\
& 0.195 \\
& 0.120 \\
& 0.070 \\
& 0.010 \\
& 0.983 \\
& 0.081\end{aligned}$ \\
\hline $\begin{array}{l}\text { Relacionadas con ETV } \\
\text { Embolia pulmonar concomitante }\end{array}$ & $23.44 \%(64)$ & $19.62 \%(124)$ & 0.193 \\
\hline
\end{tabular}

*Media ( \pm desviación estándar).

CE: consumidores de estatinas; NCE: no consumidores de estatinas; TVP: trombosis venosa profunda; EPOC: enfermedad pulmonar obstructiva crónica;

AINE: antiinflamatorios no esteroideos; ETV: enfermedad tromboembólica venosa.

molecular, $46.20 \%$ con AVK y $0.63 \%$ con NOA), con diferencia significativa ( $p=0.036)$.

La cantidad de eventos de SPT detectados durante el seguimiento fue de 18 en el grupo CE y 51 en el grupo NCE. La mediana de tiempo de seguimiento global de la cohorte fue de 15 meses (RIC: 0-38). La tabla 4 muestra los eventos detectados en el seguimiento. 
Tabla 3. Detalles sobre consumo de estatinas en el grupo de consumidores de estatinas (CE)

\begin{tabular}{|c|c|}
\hline Tipo de estatina & CE $(\mathbf{n}=\mathbf{2 7 3})$ \\
\hline Rosuvastatina & $24.91 \%(68)$ \\
$5 \mathrm{mg}$ & $22.05 \%(15)$ \\
$10 \mathrm{mg}$ & $64.70 \%(44)$ \\
$20 \mathrm{mg}$ & $13.25 \%(9)$ \\
\hline Atorvastatina & $51.28 \%(140)$ \\
$10 \mathrm{mg}$ & $52.14 \%(73)$ \\
$20 \mathrm{mg}$ & $47.86 \%(67)$ \\
\hline Simvastatina & $23.81 \%(65)$ \\
$10 \mathrm{mg}$ & $46.15 \%(30)$ \\
$20 \mathrm{mg}$ & $47.69 \%(31)$ \\
$40 \mathrm{mg}$ & $6.16 \%(4)$ \\
\hline
\end{tabular}

Tabla 4. Cantidad de eventos evaluados durante al seguimiento por grupo CE y NCE

\begin{tabular}{|l|c|c|c|}
\hline & CE (n = 273) & NCE (n = 632) & p* \\
\hline SPT & 18 & 51 & 0.412 \\
\hline RETV & 30 & 68 & 0.403 \\
\hline Fallecimiento & 120 & 322 & 0.047 \\
\hline $\begin{array}{l}\text { Mediana de } \\
\text { seguimiento a la } \\
\text { última fecha de } \\
\text { contacto, en meses }\end{array}$ & 17 (IIC 0-44) & 15 (IIC 0-36.5) & 0.177 \\
\hline $\begin{array}{l}\text { *p valor estimado de test de Log Rank. } \\
\text { CE: consumidores de estatinas; NCE: no consumidores de estatinas; SPT: síndrome } \\
\text { postrombótico; RETV: recurrencia de enfermedad tromboembólica venosa. }\end{array}$
\end{tabular}

\section{Asociación entre consumo de estatinas y síndrome postrombótico}

La densidad de incidencia de SPT en el grupo CE fue de 3.69 (IC 95\%: 2.22-6.12) por cada 100 personas/año, y de 4.68 (IC 95\%: 3.43-6.38) por cada 100 personas/año en el grupo NCE. La razón de densidad de incidencias fue de 0.79 (IC 95\%: 0.4-1.46) para los CE con respecto a los NCE. La tabla 5 presenta las incidencias acumuladas anuales para ambos grupos. No se pudo estimar la mediana de tiempo al evento porque ninguna de los dos grupos tuvo un $50 \%$ de incidencia acumulada de eventos SPT. La mediana de tiempo al desarrollo de SPT fue 9 meses, siendo de 8 meses en el grupo NCE y 11 meses en CE. En la figura 2 se presenta el gráfico de Kaplan Meier para desarrollo de SPT entre CE y NCE (log rank test: $p=0.412$ ).

La HR cruda del CE fue de 0.78 (IC 95\%: 0.43-1.41; $p=0.414)$ para el desarrollo de SPT. La HR del CE ajustada por edad, sexo, AINE, corticosteroides, inmovilidad, tratamiento anticoagulante, HTA, DBT, DLP, IRC,
Tabla 5. Incidencias acumuladas de SPT estimadas con el método de Kaplan Meier, en ambos grupos, CE y NCE

\begin{tabular}{|l|c|c|}
\hline $\begin{array}{l}\text { Incidencia acumulada de SPT } \\
\text { (meses) }\end{array}$ & $\begin{array}{c}\text { CE (\% con } \\
\text { IC 95\%) }\end{array}$ & $\begin{array}{c}\text { NCE (\% con } \\
\text { IC 95\%) }\end{array}$ \\
\hline 12 & 5.03 & 4.62 \\
$(2.54-9.85)$ & $(2.85-7.47)$ \\
\hline 24 & 7.57 & 7.9 \\
\hline 36 & $(4.22-13.38)$ & $(5.24-11.82)$ \\
\hline 48 & 10.69 & 12.58 \\
\hline 60 & $(6.05-18.52)$ & $(8.69-18.03)$ \\
\hline & 12.59 & 18.57 \\
\hline & $(7.19-21.55)$ & $(13.13-25.91)$ \\
\hline
\end{tabular}

CE: consumidores de estatinas; NCE: no consumidores de estatinas; SPT: síndrome postrombótico; IC: intervalo de confianza.

Tabla 6. Asociación entre el consumo de estatinas y el desarrollo de SPT. Modelo de regresión de Cox

\begin{tabular}{|l|c|c|c|}
\hline & HR & IC 95\% & p \\
\hline Edad, por cada aumento en 1 año & 0.99 & $(0.97-1)$ & 0.1 \\
\hline Sexo femenino & 1.56 & $(0.86-2.84)$ & 0.144 \\
\hline AINE & 0.86 & $(0.47-1.59)$ & 0.639 \\
\hline Corticosteroides & 1.18 & $(0.62-2.24)$ & 0.619 \\
\hline Inmovilidad & 3.03 & $(1.57-5.87)$ & 0.001 \\
\hline Recibió tratamiento anticoagulante & 1.1 & $(0.32-3.8)$ & 0.883 \\
\hline Enfermedad oncológica & 0.87 & $(0.42-1.78)$ & 0.702 \\
\hline CE vs. NCE cruda & 0.78 & $(0.43-1.41)$ & 0.414 \\
\hline CE vs. NCE ajustado* & 0.45 & $(0.13-1.5)$ & 0.196 \\
\hline CE vs. NCE ajustado por ${ }^{\dagger}$ PS & 0.52 & $(0.17-1.66)$ & 0.272 \\
\hline
\end{tabular}

*Ajustada por edad, sexo, AINE, corticosteroides, inmovilidad, tratamiento anticoagulante, HTA, DBT, dislipidemia, IRC, enfermedad coronaria, ACV, ICC y enfermedad oncológica.

${ }^{\dagger}$ Ajustada por edad, sexo, AINE, corticosteroides, inmovilidad, tratamiento anticoagulante, enfermedad oncológica y PS para consumo de estatinas. SPT: síndrome postrombótico; HR: hazard ratio; IC: intervalo de confianza; AINE: antiinflamatorios no esteroideos; CE: consumidores de estatinas; NCE: no consumidores de estatinas; PS: propensity score.

enfermedad coronaria, ACV, ICC y enfermedad oncológica fue de 0.45 (IC 95\%: 0.13-1.5; $p=0.196$ ) para el desarrollo de SPT. La HR del CE ajustada por edad, sexo, AINE, corticosteroides, inmovilidad, tratamiento anticoagulante, enfermedad oncológica y la PS fue de 0.52 (IC 95\%: 0.17-1.66; $p=0.272$ ) para el desarrollo de SPT. La tabla 6 presenta los resultados del análisis univariante y multivariante por regresión de Cox al SPT, con los HR estimados crudos y ajustados, con sus respectivos IC95\%. 


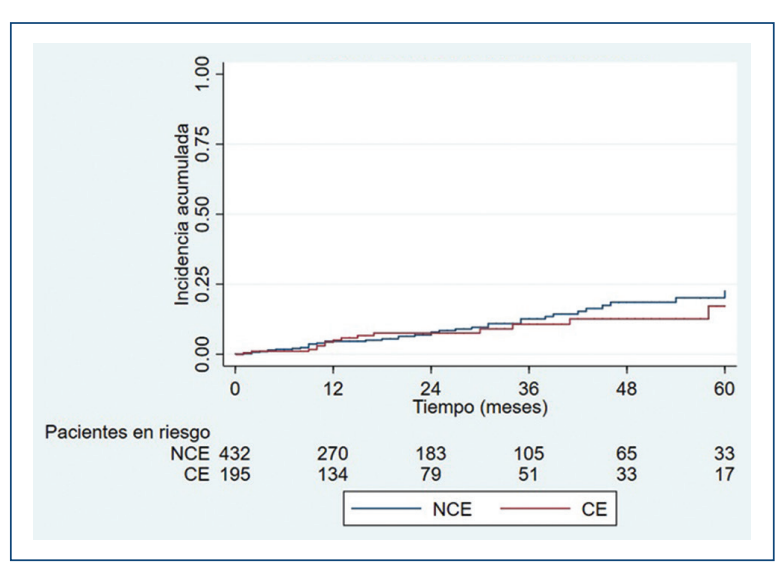

Figura 2. Incidencia acumulada de SPT estimada en función del tiempo para los pacientes con CE (rojo) y NCE (azul). SPT: síndrome postrombótico; CE: consumidores de estatinas; NCE: no consumidores de estatinas.

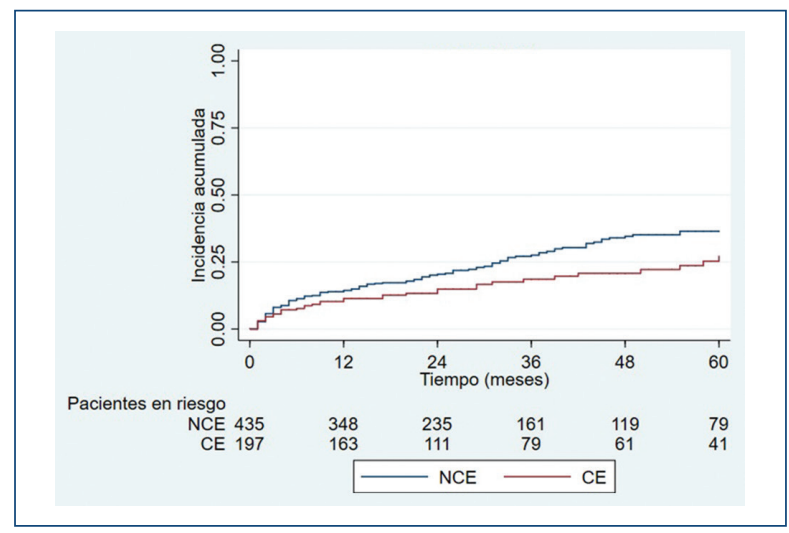

Figura 3. Mortalidad estimada con el método de Kaplan Meier en función del tiempo para los pacientes con CE (rojo) y NCE (azul).

CE: consumidores de estatinas; NCE: no consumidores de estatinas.

Considerando los eventos competitivos como análisis de sensibilidad, la sHR cruda del CE fue de 0.84 (IC 95\%: 0.46-1.51; $p=0.555$ ) para el desarrollo de SPT. La sHR del CE ajustado por edad, sexo, AINE, corticosteroides, inmovilidad, tratamiento anticoagulante, enfermedad oncológica y la PS fue de 0.45 (IC 95\%: 0.14-1.46; $p=0.184$ ) para el desarrollo de SPT.

\section{Asociación entre consumo de estatinas y fallecimiento}

La tabla 7 muestra las incidencias acumuladas por grupos, cada 12 meses. La mediana de tiempo de
Tabla 7. Incidencias acumuladas de fallecimiento, estimadas con el método de Kaplan Meier, en ambos grupos, CE y NCE

\begin{tabular}{|l|c|c|}
\hline $\begin{array}{l}\text { Incidencia acumulada } \\
\text { de fallecimiento } \\
\text { (meses) }\end{array}$ & $\begin{array}{c}\text { CE }(\% \text { con } \\
\text { IC 95\%) }\end{array}$ & $\begin{array}{c}\text { NCE }(\% \\
\text { con IC 95\%) }\end{array}$ \\
\hline 12 & $11.36(7.63-16.74)$ & $14.41(11.42-18.1)$ \\
\hline 24 & $14.86(10.39-21.04)$ & $20.45(16.79-24.77)$ \\
\hline 36 & $18.58(13.26-25.7)$ & $27.56(23.09-32.7)$ \\
\hline 48 & $20.79(14.96-28.49)$ & $34.58(29.33-40.47)$ \\
\hline 60 & $27.13(19.57-36.85)$ & $36.47(30.98-42.6)$ \\
\hline
\end{tabular}

IC: intervalo de confianza; CE: consumidores de estatinas; NCE: no consumidores de estatinas.

seguimiento al fallecimiento de la cohorte global fue de 15 meses, siendo de 15 meses en el grupo NCE y 17 meses en el de CE.

En la figura 3 se presenta el gráfico de Kaplan Meier para mortalidad entre CE y NCE (log rank test: $p=0.046)$. La HR cruda del CE para fallecimiento fue de 0.71 (IC 95\%: 0.5-0.99; $p=0.049$ ). La HR del CE ajustada por edad, sexo, AINE, corticosteroides, inmovilidad, tratamiento anticoagulante, enfermedad oncológica, score de comorbilidades de Charlson y la PS fue de 0.62 (IC 95\%: 0.4-0.98; $p=0.04$ ).

\section{Discusión}

En nuestro estudio no encontramos asociación entre el CE y el desarrollo del SPT, y se trata de un estudio pragmático, que representa el escenario más parecido al seguimiento habitual de los pacientes en el mundo real. Este tipo de diseño aumenta la validez externa, aunque tienen mayor variabilidad en sus mediciones $y$ procesos (p. ej., la adherencia, las dosis, las diferentes estatinas, las comorbilidades de los pacientes, el seguimiento irregular), y requieren mayores tamaños muestrales. Cabe destacar que la gran limitante de este trabajo radica en el baja potencia estadística basada en la baja incidencia del SPT. Por medio de estrategia múltiple de recolección se detectaron 69 casos en total, 18 en el grupo CE y 51 en el grupo de NCE, y solo 8 detectados mediante la historia clínica, pero esto podría explicar la falta de significancia estadística.

El efecto de las estatinas sobre la ETV se observó por primera vez en el estudio JUPITER ${ }^{19}$, que exploró como objetivo secundario el efecto de la rosuvastatina sobre la disminución de ETV en pacientes con proteína 
C reactiva (PCR) elevada, evidenciando una reducción del riesgo del $43 \%$ de TVP en pacientes tratados con rosuvastatina (OR: 0.57; IC 95\%: 0.37-0.86). Si bien la validez externa es limitada, motivó diferentes estudios posteriores sobre este argumento. La plausibilidad biológica de los posibles mecanismos que expliquen una asociación entre el CE y el SPT se basan en su definición y origen, tratándose del resultado de la hipertensión venosa que sigue al desarrollo de la incompetencia valvular, al reflujo y a la obstrucción venosa. La incompetencia valvular es la consecuencia del daño sufrido por las válvulas venosas en el momento del episodio trombótico agudo y la fase de recanalización venosa. En estas fases actúan diferentes componentes inflamatorios que son uno de los factores determinantes del desarrollo de SPT. Por un lado las células endoteliales microvasculares que son metabólicamente activas expresan diferentes moléculas de adhesión intracelular de clase 1 (ICAM-1), moléculas de adhesión vascular de clase 1 (VCAM-1) y menor expresión de selectina $E$ que las células endoteliales normales ${ }^{20}$. Los leucocitos circundantes, en su mayoría neutrófilos, linfocitos T, monocitos y macrófagos potencian la respuesta inflamatoria al expresar ciertas moléculas de adhesión y al secretar mediadores inflamatorios como la interleucina (IL) 1, IL-6, IL-8, P-selectina, la proteína quimiotáctica de monocitos 1 (MCP-1), factor de necrosis tumoral (TNF) beta 1 (TNF- $\beta 1$ ) y alfa (TNF- $\alpha$ ). Por otra parte, la liberación y activación de las células leucocitarias actúan sobre las células intersticiales, que como respuestas a los mediadores liberados por las células endoteliales vasculares y los leucocitos, estimulan el reclutamiento y la activación de macrófagos, fibroblastos y la expresión de diferentes proteínas del tejido conectivo, con la posterior reparación del tejido dañado. Debido a que el SPT es consecuencia de una gran reacción inflamatoria, nos parece importante destacar que las estatinas, hipolipemiantes pero con un efecto pleiotrópico y antiinflamatorio, podrían ayudar a disminuir la inflamación crónica generada al actuar sobre la regulación, la disminución en la expresión y secreción de diferentes factores proinflamatorios como ICAM-1, VCAM-1, IL-1, IL-6, IL-8, TNF- $\beta 1$ y TNF- $\alpha^{6,21}$. De esta manera, al actuar sobre estos diferentes componentes de la inflamación podrían disminuir el desarrollo del SPT en los pacientes que hayan tenido una TVP8.

En esta misma línea, San Norberto, et al. realizaron un ensayo con 234 pacientes con diagnóstico de ETV sin TEP ${ }^{22}$, y como objetivo secundario evaluaron el efecto de las estatinas sobre el grado de SPT. El grupo de pacientes tratados con rosuvastatina presentó una reducción de los niveles de PCR y de la incidencia de SPT (38.3 vs. $48.5 \% ; p=0.019$ ), luego de tres meses de seguimiento. A diferencia de nuestro estudio, en este tenían menor cantidad de comorbilidades y nosotros incluimos pacientes con TEP concomitante; la menor carga de ETV podría favorecer el efecto de la rosuvastatina y explicar sus resultados sin significancia estadística, pero con misma dirección de la asociación (estatinas como protectoras), en consistencia con nuestros resultados (HR cruda: 0.78; IC 95\%: 0.43-1.41; $p=0.414)$.

Con respecto a detección del SPT, en el estudio de Ghanima, et al. ${ }^{23}$ y en el de San Norberto, et al. se utilizó entrevista clínica estructurada con escala de Villalta (considerando SPT una puntuación > 5 o si presentaba úlcera venosa ${ }^{22}$, mientras que en nuestro estudio se identificaron por medio de una adaptación del cuestionario de Villalta ${ }^{16}$ y por revisión sistemática de HCE. Si bien la evaluación telefónica es más factible y práctica, es probable que solo detecte los casos graves o sintomáticos de SPT. Esta diferencia en los métodos de detección podría explicar la diferencia observada entre el SPT en nuestra cohorte del $7.62 \%$, siendo inferior comparada con estos estudios, donde rondan el $40 \%$. Adicionalmente, debido a que la frecuencia de SPT en nuestro estudio fue menor a la esperada, la ausencia de diferencias estadísticamente significativas podría deberse a falta de potencia para detectar diferencias entre los CE y NCE.

En referencia al $\mathrm{CE}$, en nuestra cohorte la estatina de mayor frecuencia de uso fue la atorvastatina, $51.28 \%$ en una dosis de $10 \mathrm{mg}$ por día, en contraste con otros estudios que describen el uso de rosuvastatina ${ }^{24,25}$.

Nuestros resultados con respecto a la recurrencia de enfermedad tromboembólica venosa (RETV) se asemejan al estudio de Delluc, et al. ${ }^{26}$, una cohorte prospectiva de 432 pacientes con un primer evento de ETV. En el análisis multivariante el riesgo de RETV asociado con la exposición a estatinas fue de 1.02 (IC 95\%: 0.362.91) mientras que en nuestra cohorte la $\mathrm{HR}$ del $\mathrm{CE}$ ajustada fue de 0.74 (IC 95\%: 0.37-1.49). Si bien ambos estudios fueron no estadísticamente significativos, ellos seleccionaron una población con menos comorbilidades que la nuestra, ya que excluyen la ETV secundaria. Asimismo, ellos realizaron una evaluación más activa del consumo de fármacos hipolipemiantes, tuvieron en cuenta el tiempo de anticoagulación y el sitio de trombosis, aspectos que nosotros no pudimos evaluar.

Nuestros resultados del análisis de sensibilidad fueron consistentes con el análisis principal y esta 
concordancia aumenta la robustez y consistencia de nuestros hallazgos.

\section{Agradecimientos}

A todas las personas que colaboraron en el proyecto brindando ayuda técnica como asistencia en la redacción del manuscrito, y a los jefes (Dr. Javier Pollán y Dr. Bernardo Martínez), que brindaron apoyo general. Este proyecto representa la tesis de Maestría de Investigación Clínica del Instituto Universitario Hospital Italiano del autor principal y de correspondencia.

\section{Financiamiento}

La presente investigación no ha recibido ninguna beca específica de agencias de los sectores público, comercial o sin ánimo de lucro.

\section{Conflictos de intereses}

La presente investigación no ha recibido ayudas específicas provenientes de agencias del sector público, sector comercial o entidades sin ánimo de lucro.

\section{Responsabilidades éticas}

Protección de personas y animales. Los autores declaran que los procedimientos seguidos se conformaron a las normas éticas del comité de experimentación humana responsable y de acuerdo con la Asociación Médica Mundial y la Declaración de Helsinki.

Confidencialidad de los datos. Los autores declaran que han seguido los protocolos de su centro de trabajo sobre la publicación de datos de pacientes.

Derecho a la privacidad y consentimiento informado. Los autores han obtenido el consentimiento informado de los pacientes y/o sujetos referidos en el artículo. Este documento obra en poder del autor de correspondencia.

\section{Bibliografía}

1. Gabriel Botella F, Labiós Gómez M, Portolés Reparaz O, Cabanes Vila J. Nuevos avances en el conocimiento del síndrome postrombótico. An Med Interna. 2003;20(9).
2. ISTH Steering Committee for World Thrombosis Day. Thrombosis: a major contributor to the global disease burden. J Thromb Haemost. 2014;12(10):1580-90.

3. Prandoni $P$, Lensing AW, Cogo A, Cuppini S, Villalta S, Carta M, et al. The long-term clinical course of acute deep venous thrombosis. Ann Intern Med. 1996;125(1):1-7.

4. Goldhaber SZ, Bounameaux H. Pulmonary embolism and deep vein thrombosis. Lancet. 2012;379(9828):1835-46.

5. Suematsu M, Ishimura Y, Schmid-Schönbein GW. Temporal and spatial dissociation between tissue leukocyte recruitment and cell injury in the rat postischemic skeletal muscle microcirculation. Pathophysiology. 1994:1:211.

6. Wassmann S, Nickenig G. Interrelationship of free oxygen radicals and endothelial dysfunction-modulation by statins. Endothelium. 2003;10(1):23-33.

7. Huacuja Alvarez F, Gómez Duque M, Ortiz Vargas JC, Soberanes Velázquez B, Arévalo Moreno V, Morales Villegas E, et al. Efecto de las estatinas más allá del colesterol. Rev Endocrinol Nutr. 2006;14(2):73-88.

8. Rodriguez AL, Wojcik BM, Wrobleski SK, Myers DD Jr, Wakefield TW, Diaz JA. Statins, inflammation and deep vein thrombosis: a systematic review. J Thromb Thrombolysis. 2012;33(4):371-82.

9. Luna D, Otero P, López Osornio A, Ríos E, Pedernera FA, Gómez A et al. Implementación de una historia clínica electrónica ambulatoria: El Proyecto Itálica [Internet]. 6.ํ Simposio de Informática en Salud - 32 JAllO 2003. Disponible en: https://www.academia.edu/23049560/Implementaci\%C3\%B3n_de_una_Historia_Cl\%C3\%ADnica_Electr\%C3\%B3nica_Ambulatoria_Proyecto_ITALICA

10. Plazzotta F, Luna D, González Bernaldo de Quirós F. Sistemas de información en salud: Integrando datos clínicos en diferentes escenarios y usuarios. Rev Peru Med Exp Salud Publica. 2015;32(2):343-51.

11. SNOMED International [Internet]. Disponible en: http://www.snomed.org/ snomed-ct

12. Franco M, Giussi Bordoni MV, Otero C, Landoni MC, Benitez S, Borbolla D, et al. Problem oriented medical record: Characterizing the use of the problem list at Hospital Italiano de Buenos Aires. Stud Health Technol Inform. 2015;216:877.

13. Luna D, Franco M, Plaza C, Otero C, Wassermann S, Gambarte ML, et al. Accuracy of an electronic problem list from primary care providers and specialists. Stud Health Technol Inform. 2013;192:417-21.

14. Plazzotta F, Otero C, Luna D, de Quiros FGB. Natural language processing and inference rules as strategies for updating problem list in an electronic health record. Stud Health Technol Inform. 2013;192:1163.

15. ATC/DDD Index 2020 [Internet]. WHO Collaborating Centre for Drug Statistics Methodology. Disponible en: https://www.whocc.no/atc_ddd_index/

16. Posadas-Martínez L, Pazo V, Vázquez F, Arbelbide J, Waisman G, Bernaldo de Quirós F, et al. Síndrome postrombótico: validación de una escala telefónica como herramienta diagnóstica. Rev Argent Med. 2014;2:33-8.

17. Zhang X, Zhang MJ, Fine J. A proportional hazards regression model for the subdistribution with right-censored and left-truncated competing risks data. Stat Med. 2011;30(16):1933.

18. Fine JP, Gray RJ. A proportional hazards model for the subdistribution of a competing risk. J Am Stat Assoc. 1999;94(446):496-509.

19. Rosenstein R, Parra D. Rosuvastatin. C-reactive protein, LDL cholesterol, and the JUPITER trial. Lancet. 2009;374(9683):25.

20. Weyl A, Vanscheidt W, Weiss JM, Peschen M, Schopf E, Simon J. Expression of the adhesion molecules ICAM-1, VCAM-1, and E-selectin and their ligands VLA-4 and LFA-1 in chronic venous leg ulcers. J Am Acad Dermatol. 1996;34(3):418-23.

21. Mennickent CS, Bravo DM, Calvo MC, Avello LM. Pleiotropic effects of statins. Rev Med Chile. 2008;136(6):775-82.

22. San Norberto EM, Norberto EMS, Gastambide MV, Taylor JH, García-Saiz I, Vaquero C. Effects of rosuvastatin as an adjuvant treatment for deep vein thrombosis. Vasa. 2016;45(2):133-40.

23. Ghanima W, Kleven IW, Enden T, Rosales A, Wik HS, Pederstad L, et al Recurrent venous thrombosis, post-thrombotic syndrome and quality of life after catheter-directed thrombolysis in severe proximal deep vein thrombosis. J Thromb Haemost. 2011;9(6):1261-3.

24. Glynn RJ, Danielson E, Fonseca FAH, Genest J, Gotto AM Jr, Kastelein JJP, et al. A randomized trial of rosuvastatin in the prevention of venous thromboembolism. N Engl J Med. 2009;360(18):1851-61.

25. Kunutsor SK, Seidu S, Khunti K. Statins and primary prevention of venous thromboembolism: a systematic review and meta-analysis. Lancet Haematol. 2017;4(2):e83-93.

26. Delluc A, Tromeur C, Le Moigne E, Nowak E, Mottier D, Le Gal G, et al. Lipid lowering drugs and the risk of recurrent venous thromboembolism. Thromb Res. 2012;130(6):859-63. 